\title{
A generalized model for compact stars
}

\author{
Abdul Aziz ${ }^{1, \mathrm{a}}$, Saibal Ray ${ }^{2, \mathrm{~b}}$, Farook Rahaman ${ }^{3, \mathrm{c}}$ \\ ${ }^{1}$ Department of Physics, Bodai High School (H.S.), Bodai, Amdanga, North 24 Parganas, Kolkata, West Bengal 700126, India \\ ${ }^{2}$ Department of Physics, Government College of Engineering and Ceramic Technology, Kolkata, West Bengal 700010, India \\ ${ }^{3}$ Department of Mathematics, Jadavpur University, Kolkata, West Bengal 700032, India
}

Received: 17 March 2016 / Accepted: 12 April 2016 / Published online: 2 May 2016

(C) The Author(s) 2016. This article is published with open access at Springerlink.com

\begin{abstract}
By virtue of the maximum entropy principle, we get an Euler-Lagrange equation which is a highly nonlinear differential equation containing the mass function and its derivatives. Solving the equation by a homotopy perturbation method we derive a generalized expression for the mass which is a polynomial function of the radial distance. Using the mass function we find a partially stable configuration and its characteristics. We show that different physical features of the known compact stars, viz. Her $X-1, R X J$ 1856-37, $S A X J$ (SS1), SAX J (SS2), and PSR J 1614-2230, can be explained by the present model.
\end{abstract}

\section{Introduction}

To find an analytical solution of nonlinear equations has always been a great challenge in relativistic astrophysics as well as cosmology. Considering different methods and approximations mathematicians and relativists attempted to find the suitable interior solutions of physical interest. It is in this aspect seen that the homotopy perturbation method (HPM) is a powerful yet simple tool to solve several highly nonlinear equations with the least number of assumptions as can be seen in the literature [1-13].

It is interesting to note that in one of his earlier works $\mathrm{He}$ [4] proposed a coupling method of a homotopy technique and a perturbation technique to solve a nonlinear equation. In contrast to the traditional perturbation methods, the proposed method by He did not require any small perturbation in the equation to obtain an effective and simple solution. Thereafter several researchers have employed the HPM in the diverse fields of mathematics and physics [14-18].

For special mention in the field of astrophysics, it is observed that by using the MIT bag model, Rahaman et

\footnotetext{
a e-mail: azizmail2012@gmail.com

be-mail: saibal@associates.iucaa.in

ce-mail: rahaman@associates.iucaa.in
}

al. [19] obtained a deterministic model for a strange star where they considered a mass polynomial and analyzed critically all the physical properties from the model. However, their model was unable to explain the physical properties up to $6 \mathrm{~km}$ from the center of the spherical system.

Deb et al. [20] attempted to find a new class of solutions relating to a radiation model by using the HPM. The spherical distribution was considered to be made of a perfect fluid where radiation is along the radially outward direction of the system. Here we have employed the HPM as a new tool for astrophysical systems which via the mass polynomial facilitates solving of the field equations. A set of interior solutions has been found on the basis of the equation of state in the form $p=\frac{1}{3} \rho$, where $p$ and $\rho$ are the radial pressure and density of the matter distribution, with the requirement that the radiation model is consistent with the Schwarzschild metric. It is observed that the set of solutions thus developed provides a new metric to explain strange stars, which, however, seems to be not free from a singularity.

In another work Rahaman et al. [21] employed the HPM for a spherically symmetric system of a radiating star which was shown to suffer from an instability problem. It is observed from the mass function that it was possible to explain the features of an $E 0$ type brown dwarf star with $0.065 M_{\odot}$ (i.e. $45227 \mathrm{~km}$ ) whereas the stability of the brown dwarf could not be explained which suffers from an instability within the region of the core radius $41800 \mathrm{~km}$. However, in the present investigation we propose a general mass function and use it for different cases.

Therefore the motivation of the present work is to provide a general model for compact stars by employing a polynomial parameter $n$ and to find the different features of compact stars with this mass function. We derive expressions for the density, pressure, redshift etc. and have shown that several known compact stars, e.g. Her $X-1, R X J 1856-37$, $S A X J$ (SS1), SAX J (SS2), and PSR J 1614-2230, can be well explained by this model. 
The plan of the investigation is as follows: in Sect. 2 we have calculated the mass of the system by using the maximum entropy principle (MEP), and thereafter by using the HPM we have obtained a suitable expression for the mass. We have reformatted Einstein's field equations by using the mass function and obtain solutions for the system in Sect. 3. Also in Sect. 3 we have explored different physical features, viz. the density and mass, pressure and anisotropy, stability, energy conditions, TOV equations, compactness, redshift, etc. with elaborate discussion and a comparative study of the available data from observation with that of the predicted values. In Sect. 4 we have made some concluding remarks regarding different aspects of the present model.

\section{Spherically symmetric spacetime and the mass function}

\subsection{Maximum entropy principle}

Let us consider the metric of a system which has spherical symmetry as follows

$$
\begin{aligned}
\mathrm{d} s^{2}= & -g_{t t}(r) \mathrm{d} t^{2}+\left(1-\frac{2 m(r)}{r}\right)^{-1} \mathrm{~d} r^{2} \\
& +r^{2}\left(\mathrm{~d} \theta^{2}+\sin ^{2} \theta \mathrm{d} \phi^{2}\right)
\end{aligned}
$$

where $m(r)$ is the mass distribution of the spherically symmetric body and $g_{t t}(r)$ is the time-time component of the metric. These are functions of the radial coordinate $r$ only. Also we consider a linear equation of state for the system with thermal radiation given by

$p_{r}=\frac{1}{3} \rho$,

where $p_{r}$ and $\rho$ are the radial pressure and density of the matter distribution. Compatible with the spherically symmetry, we assume the general energy momentum tensor to be

$T_{v}^{\mu}=\left(\rho+p_{r}\right) u^{\mu} u_{v}-p_{r} g_{v}^{\mu}+\left(p_{t}-p_{r}\right) \eta^{\mu} \eta_{v}$

with

$u^{\mu} u_{\mu}=-\eta^{\mu} \eta_{\mu}=1$

The energy density and entropy density take the forms for locally measured temperature $T$ [22] as follows:

$\rho=b T^{4}$

$s=\frac{4}{3} b T^{3}$, where $b$ is a constant of order unity (in Planck units $G=c=$ $h=k=1$ ), on the assumption that the number of species of radiation is of order unity [23].

For the matter distribution up to radius $r \leq R$, the total entropy is given by

$S=4 \pi \int_{0}^{R} s(r)\left(1-\frac{2 m(r)}{r}\right)^{-1 / 2} r^{2} \mathrm{~d} r$

which is simplified as

$S=(4 \pi)^{\frac{1}{4}} \alpha \int_{0}^{R} L \mathrm{~d} r$

where the Lagrangian $L$ is given by

$L=\left(m^{\prime}\right)^{\frac{3}{4}}\left[1-\frac{2 m(r)}{r}\right]^{-\frac{1}{2}} r^{\frac{1}{2}}$.

Here, $s=\alpha(\rho)^{\frac{3}{4}}, \alpha=\frac{4}{3} b^{\frac{1}{4}}$, and the Einstein equations yield

$\rho=\frac{m^{\prime}(r)}{4 \pi r^{2}}$.

Also, we have the Euler-Lagrange equation

$\frac{\mathrm{d}}{\mathrm{d} r}\left(\frac{\partial L}{\partial m^{\prime}}\right)-\frac{\partial L}{\partial m}=0$,

which can be reduced to

$m^{\prime}+m^{\prime \prime} m-\frac{1}{2} m^{\prime \prime} r-\frac{2}{3}\left(m^{\prime}\right)^{2}-\frac{4 m m^{\prime}}{r}=0$.

We see that the equation contains the mass, its derivatives, and products of them. So it will be very difficult to get an exact solution for this highly nonlinear differential equation. Therefore we use a homotopy perturbation method to solve the equation and thus we get an approximate solution for the mass.

\subsection{Homotopy perturbation method}

In the HPM we consider the linear and nonlinear parts of the differential equation separately. Then we build the homotopy structure [4] perturbed with a parameter, say $\epsilon$, as follows:

$L(m)-L\left(m_{0}\right)+\epsilon L\left(m_{0}\right)+\epsilon[$ nonlinear part $]=0$.

For the present problem we take the linear part $L(m)$ as $\frac{\mathrm{d} m}{\mathrm{~d} r}$ and the rest of the terms as the nonlinear part. So the above equation becomes

$m^{\prime}-m_{0}^{\prime}+\epsilon\left[m_{0}^{\prime}+m m^{\prime \prime}-\frac{1}{2} m^{\prime \prime} r-\frac{2}{3}\left(m^{\prime}\right)^{2}-\frac{4 m m^{\prime}}{r}\right]=0$, 
where $m_{0}$ is the initial solution of the linear part of the equation. Now we assume the general solution

$m=m_{0}+\epsilon m_{1}+\epsilon^{2} m_{2}+\epsilon^{3} m_{3}+\cdots$.

By varying the value of $\epsilon$ from 0 to 1 we shall get the desired solution of the nonlinear equation (10).

Since we are looking for a more general expression for the mass we assume the initial solution as $m_{0}=a r^{n}$ where we choose ' $a$ ' as model parameter, $r$ as radial distance; $n$ is an index assumed to have any value.

Substituting this in Eq. (12) and equating coefficients of the different orders $\epsilon$, we get the solution for the mass up to a second order correction as

$m=m_{0}+m_{1}+m_{2}$.

Again, adding the correction terms to the initial solution we get the general expression for the mass as

$$
\begin{aligned}
m= & {\left[\frac{(n-1)^{2}}{4} a r^{n}+\frac{n(5-n)}{(n-1)} A a r^{n-1}\right.} \\
& \left.+\frac{n(15-n)(2 n-3)}{3(2 n-1)} a^{2} r^{2 n-1}\right] \\
& +\left[\frac{n(15-n)\left(53 n-7 n^{2}-18\right)}{9(3 n-2)(2 n-1)} a^{3} r^{3 n-2}+B\right],
\end{aligned}
$$

where $A$ and $B$ are the constants of integration.

Here we get the mass as a polynomial function of the radial distance with different powers of $n$. Now this equation can be utilized to get a different expression of the mass for different values of $n$. It is the mass function here which actually governs different features of the stars. So, primarily the goal of the paper is to develop a generalized mass function using the HPM. At a first glance, we observe that the mass function becomes undefined for $n=1$ and some fractional values of $n$, e.g. $1 / 2,2 / 3$. The values of the $n$ should be such that the resultant mass function be a well-defined, positive, and increasing function of the radial distance. For this purpose we need to check the physical validity of the mass using the real values (integer or non-integer) of $n$. In the present model we therefore consider only those integer values of $n$ which show physical validity of the mass and other basic features of the stars.

We begin our investigation by taking integer values of $n$ as follows:

(i) For $n=0$, we find that mass is independent of the radial distance i.e. it is constant in nature. But variation of the mass of the star is mainly responsible to all the interstellar phenomena.

(ii) For $n=1$, the mass function is undefined. (iii) For $n=2$, mass is a well-defined function of the radial distance.

Hence the mass function (15), under the above restrictive values of $\mathrm{n}$, takes the form

$m(r)=\frac{1}{4} a r^{2}+\frac{26}{9} a^{2} r^{3}+\frac{130}{9} a^{3} r^{4}+6 A a r+B$.

Now, we use the boundary conditions

$m(0)=0, \quad m^{\prime}(R)=0$,

with

$m^{\prime}(r)=\frac{1}{2} a r+\frac{26}{3} a^{2} r^{2}+\frac{520}{9} a^{3} r^{3}+6 A a$,

$R$ being the radius of the sphere.

Thus, we get

$A=-\frac{R}{108}\left(9+156 a R+1040 a^{2} R^{2}\right)$

and

$B=0$.

Substituting $A$ and $B$ in Eq. (16), we get

$$
\begin{aligned}
m(r)= & \frac{1}{4} a r^{2}+\frac{26}{9} a^{2} r^{3}+\frac{130}{9} a^{3} r^{4} \\
& -\frac{a R}{18}\left(9+156 a R+1040 a^{2} R^{2}\right) r .
\end{aligned}
$$

To explore different features of a star we use this mass function in Sect. 3.

\section{Einstein's field equations and the physical parameters of a compact star}

The Einstein field equations for the matter distribution given in Eq. (3) can be written as

$$
\begin{aligned}
& \frac{2 m^{\prime}}{r^{2}}=8 \pi \rho, \\
& \frac{2 m}{r^{3}}-\left(1-\frac{2 m}{r}\right) \frac{g_{t t}^{\prime}}{g_{t t}} \frac{1}{r}=-\frac{8 \pi}{3} \rho, \\
& \quad-\left(1-\frac{2 m}{r}\right)\left[\frac{1}{2} \frac{g_{t t}^{\prime \prime}}{g_{t t}}-\frac{1}{4}\left(\frac{g_{t t}^{\prime}}{g_{t t}}\right)^{2}+\frac{1}{2 r} \frac{g_{t t}^{\prime}}{g_{t t}}\right] \\
& \quad-\left(\frac{m}{r^{2}}-\frac{m^{\prime}}{r}\right)\left(\frac{1}{r}+\frac{1}{2} \frac{g_{t t}^{\prime}}{g_{t t}}\right)=-8 \pi p_{t} .
\end{aligned}
$$

From Eq. (22) we get the density

$$
\rho=\frac{m^{\prime}}{4 \pi r^{2}}
$$


Using Eq. (18) we get an expression for the density:

$\rho=\frac{1}{4 \pi r^{2}}\left[\frac{520}{9} a^{3}\left(r^{3}-R^{3}\right)+\frac{26}{3} a^{2}\left(r^{2}-R^{2}\right)+\frac{a}{2}(r-R)\right]$.

Again, by using Eq. (2) we solve Eq. (23) for $g_{t t}$ as follows:

$g_{t t}=K \frac{\exp \int \frac{4}{3(r-2 m)}}{r(r-2 m)^{1 / 3}}$

where $K$ is a constant.

It can be noted that the exponential term cannot be evaluated analytically. So we have to make approximations to evaluate it. Also to find the constant $K$ we need boundary conditions. Actually we do not need to calculate the proper form of $g_{t t}$ in terms of the radial distance as we are only interested in finding different features such as density, pressure, etc. By intuition we see that these features are derivable from the Einstein field equations and the mass function. This is because the Einstein field equations contain ratios of $g_{t t}^{\prime}$, $g_{t t}^{\prime \prime}, g_{t t}$.

The total mass of the system is given by

$M=4 \pi \int_{0}^{R} \rho(r)\left(1-\frac{2 m(r)}{r}\right)^{-1 / 2} r^{2} \mathrm{~d} r$.

We consider $\frac{2 m(r)}{r}<1$ and simplifying the above integration we get the expression for the total mass as

$M \approx m(R)+\int_{0}^{R} \frac{m(r) m^{\prime}(r)}{r} \mathrm{~d} r$.

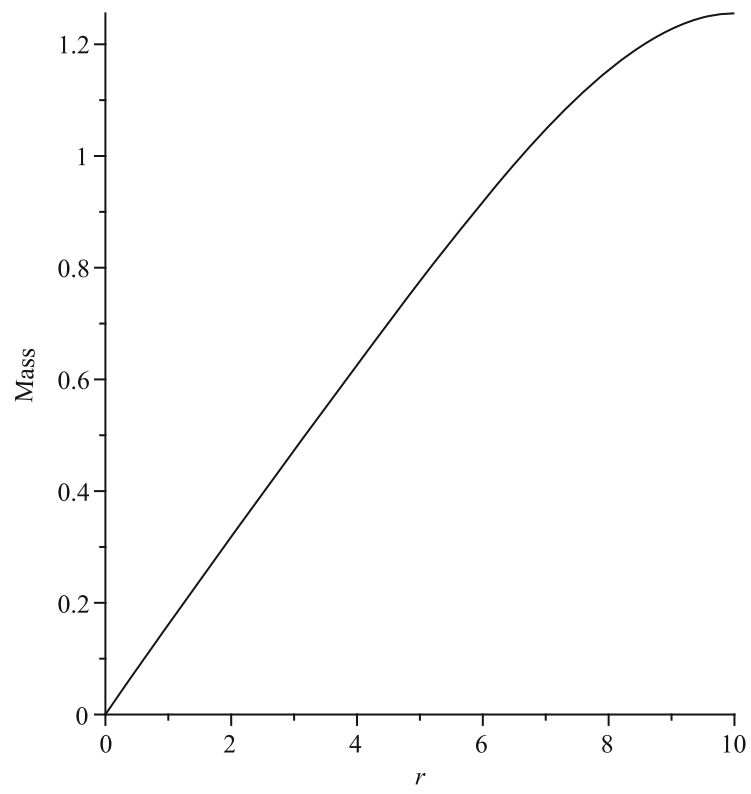

3.1 Mass and density

We begin our calculations for the group of stars of $E, M, K, G$ type etc. Calculations for these stars show that the nature of the tangential pressure is negative in nature which is against the regularity condition for a perfect fluid. Also it is observed that these stars suffer from instability.

While investigating a compact star we find that there are some specific values of $R$ and ' $a$ ' for which our model could describe them. For example, we try to explain features of a compact star, namely $\mathrm{Her} X-1$, of radius $6.7 \mathrm{~km}$ and mass of 0.9824 solar mass. We see that for the model parameter $R=10 \mathrm{~km}$ our model could describe all the features of the compact star. For this purpose we find a suitable value of the parameter ' $a$ ', which amounts to $-0.01860326472 \mathrm{~km}^{-1}$. The variations of the mass and the density are shown in Fig. 1 . We see that the mass and density are positive and they are decreasing functions.

\subsection{Pressure and anisotropy}

Using Eqs. (2) and (26) we get an expression for the radial pressure,

$p_{r}=\frac{1}{12 \pi r^{2}}\left[\frac{520}{9} a^{3}\left(r^{3}-R^{3}\right)+\frac{26}{3} a^{2}\left(r^{2}-R^{2}\right)+\frac{a}{2}(r-R)\right]$.

From Eq. (24) we obtain the expression for the tangential pressure

$p_{t}=\frac{1}{24 \pi r^{2}}\left[m^{\prime \prime} r+4 m^{\prime} \frac{\left(m^{\prime} r+3 m\right)}{(3 r-6 m)}\right]$.

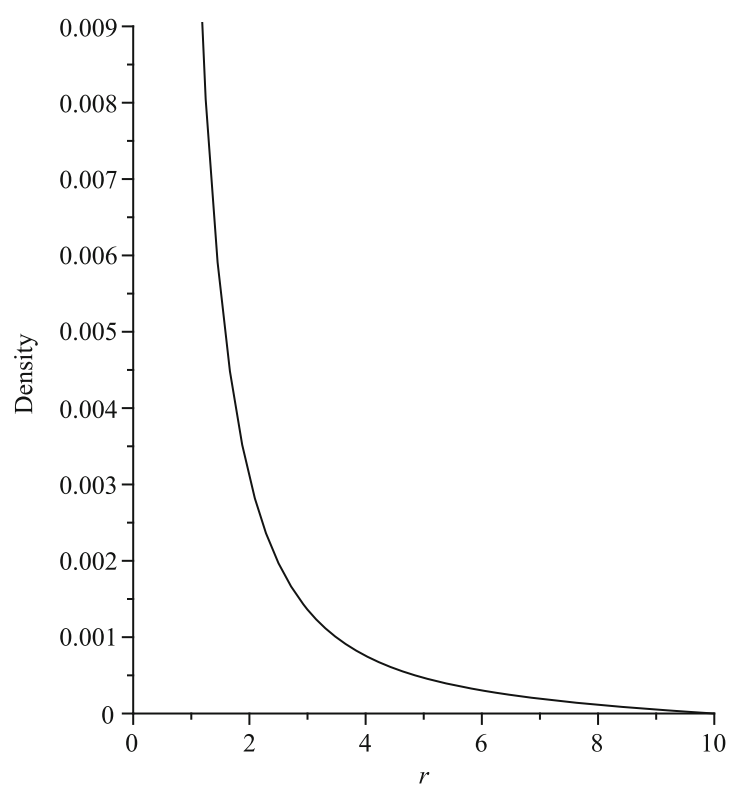

Fig. 1 Variation of mass (left panel) and density (right panel) as a function of radial distance $r$ for $a=-0.01860326472 \mathrm{~km}^{-1}$ and $R=10 \mathrm{~km}$ 

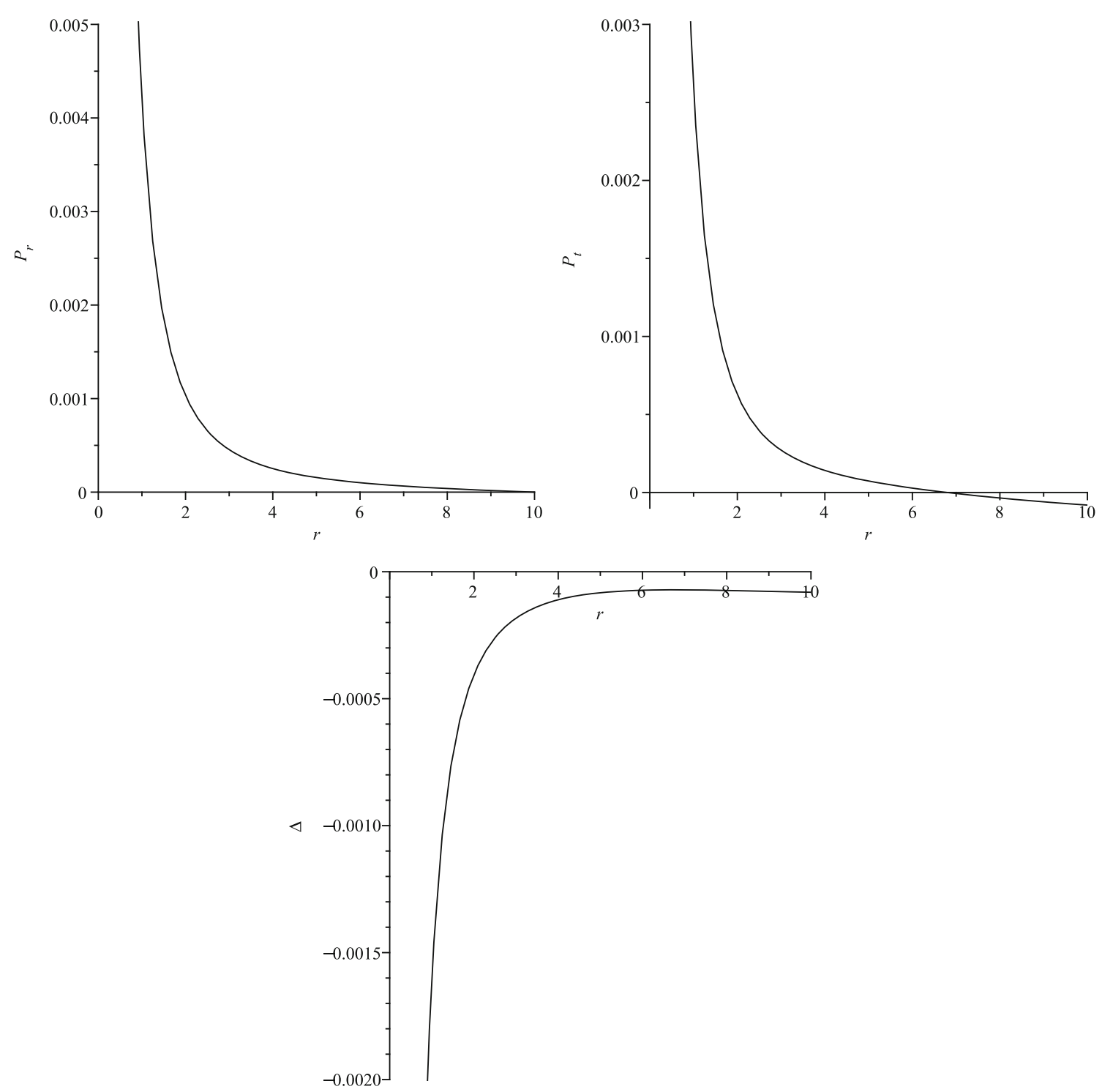

Fig. 2 Variation of radial pressure (upper left panel), tangential pressure (upper right panel) and anisotropy (lower panel) as a function of radial distance $r$ for $a=-0.01860326472 \mathrm{~km}^{-1}$ and $R=10 \mathrm{~km}$

From Fig. 2 we see that the radial pressure of the system are positive and decreasing functions of the radial distance whereas the tangential pressure switches sign at $r=6.788$ $\mathrm{km}$. Now for a perfect fluid, positivity of the pressure and the decreasing nature of the pressure are the criteria to hold good. Therefore we require the maximum allowed radius for the star to be $6.788 \mathrm{~km}$. Also we find that the total mass of star for this radius is 1.02 solar mass. These data almost match with the observed data for $\mathrm{Her} X-1$. In the following sections we shall show the variations of different features of the system by graphs.

The anisotropy for the perfect fluid is $\Delta=\left(p_{t}-p_{r}\right)$, plotted against $r$ in Fig. 2. We see that $\Delta$ is negative for any $r$. So, the anisotropic force is inward in this self-gravitating system.

\subsection{Stability condition}

We shall use Herrera's approach [24] [usually known as the concept of cracking (or overturning)] to check potential instability or stability of our configuration. According to Herrera's theorem, the region for which $v_{s t}^{2}-v_{s r}^{2}<0$ is a stable region. We observe from Fig. 3 that $v_{s t}^{2}-v_{s r}^{2}<0$ in the region for which $r<6.788 \mathrm{~km}$. This implies that our configuration is potentially stable within this region. The radial and tangential sound speeds are defined as $v_{s i}^{2}=\frac{\mathrm{d} p_{i}}{\mathrm{~d} \rho}$ (Fig. 3). 

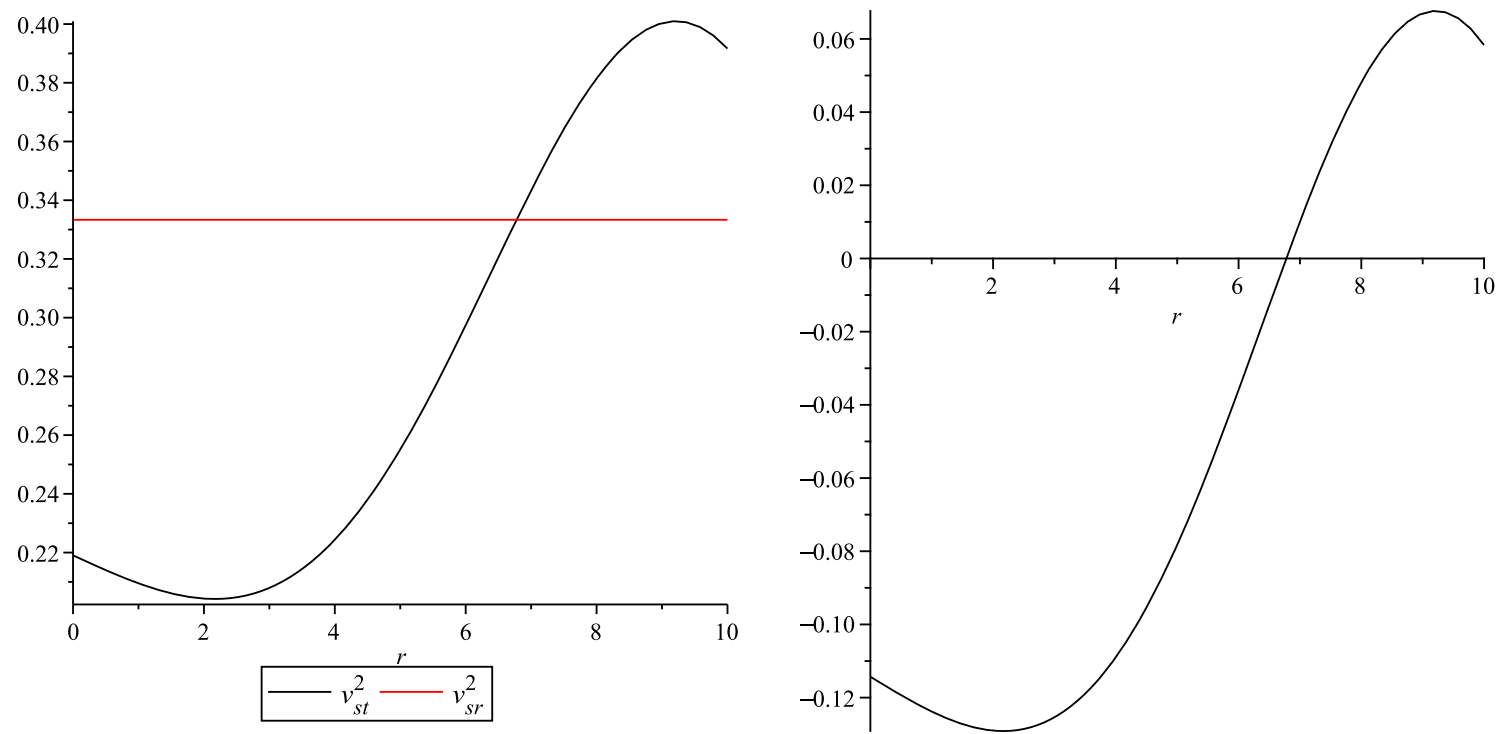

Fig. 3 Sound speeds (left panel) and difference of square of sound speeds (right panel) as a function of radial distance $r$ for $a=$ $-0.01860326472 \mathrm{~km}^{-1}$ and $R=10 \mathrm{~km}$
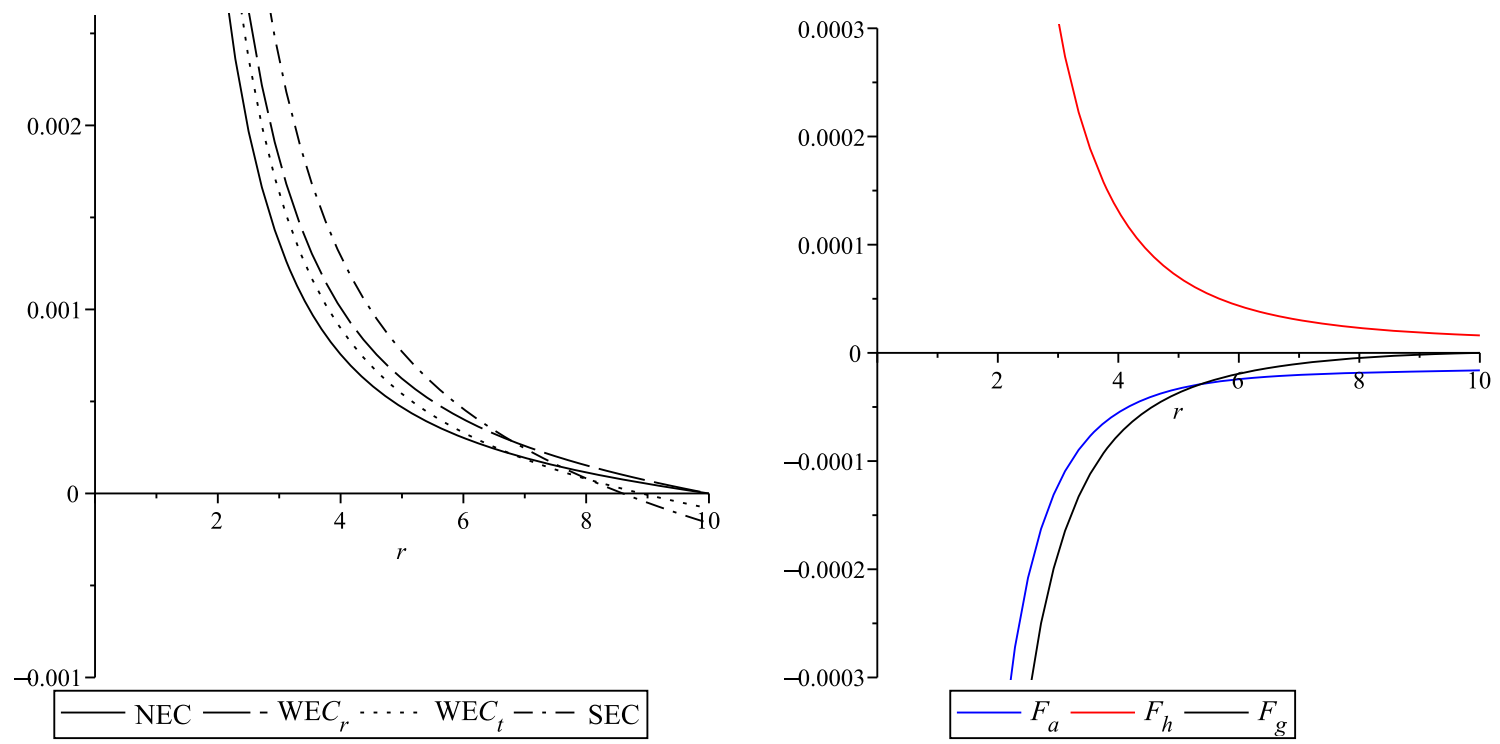

Fig. 4 Energy conditions (left panel) and different forces (right panel) as a function of radial distance $r$ for $a=-0.01860326472 \mathrm{~km}^{-1}$ and $R=10 \mathrm{~km}$

\subsection{Energy conditions}

We shall now check the energy conditions for the present model for each case:

(i) NEC: $\rho+p_{r} \geq 0, \rho+p_{t} \geq 0$,

(ii) WEC: $\rho+p_{r} \geq 0, \rho \geq 0, \rho+p_{t} \geq 0$,

(iii) SEC: $\rho+p_{r} \geq 0, \rho+p_{r}+2 p_{t} \geq 0$.

We see from Fig. 4 that different energy conditions in our model are satisfied throughout the region up to approximately $8.5 \mathrm{~km}$.

\subsection{TOV equation}

The relativistic Tolman-Oppenheimer-Volkoff (TOV) equation can be written in the following form:

$-\frac{M_{G}\left(\rho+p_{r}\right)}{r^{2}} e^{\frac{\lambda-v}{2}}-\frac{\mathrm{d} p_{r}}{\mathrm{~d} r}+\frac{2}{r}\left(p_{t}-p_{r}\right)=0$,

where $M_{G}=M_{G}(r)$ is the effective gravitational mass within the sphere up to radius $r$, given by

$M_{G}(r)=\frac{1}{2} r^{2} e^{\frac{v-\lambda}{2}} v^{\prime}$ 

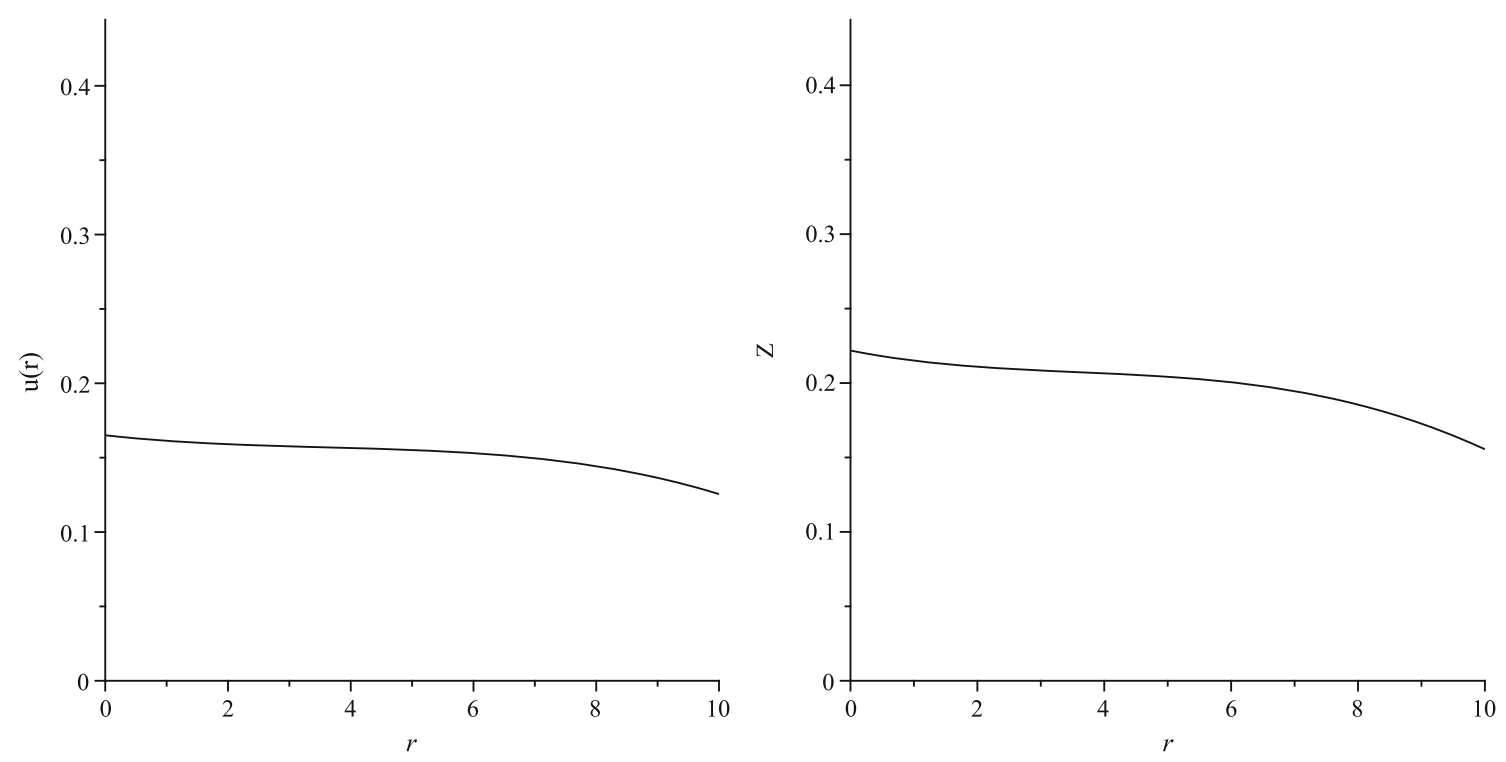

Fig. 5 Variation of compactness (left panel) and redshift (right panel) as a function of radial distance for $a=-0.01860326472 \mathrm{~km}^{-1}$ and $R=10$ $\mathrm{km}$

It is derived from the Tolman-Whittaker formula and the Einstein-Maxwell equations.

The above equation indicates that the equilibrium situation is achieved for fluid elements subject to gravitational and hydrostatic forces, and forces due to anisotropy. Thus the above equation assumes the form

$F_{g}+F_{h}+F_{a}=0$,

where

$$
\begin{aligned}
& F_{g}=-\frac{2 \rho}{3} \frac{g_{t t}^{\prime}}{g_{t t}}, \\
& F_{a}=\frac{2 \Delta}{r}, \\
& F_{h}=-\frac{\rho^{\prime}}{3} .
\end{aligned}
$$

The profiles of $F_{g}, F_{h}$, and $F_{a}$ for the sources are shown in Fig. 4. It is to be noted that $\Delta<0$ implies an attractive force, which means a hydrostatic force produces a repulsive force and essentially this stabilizes the system from collapsing due to the attractive forces $F_{a}$ and $F_{g}$. These profiles of three forces indicate that our model provides a stable configuration.

\subsection{Compactness and redshift}

The compactness of a star is defined by $u=\frac{m(r)}{r}$. By virtue of Eq. (21), this expression reduces to

$$
\begin{aligned}
u(r)= & \frac{1}{4} a r+\frac{26}{9} a^{2} r^{2}+\frac{130}{9} a^{3} r^{3} \\
& -\frac{a R}{18}\left(9+156 a R+1040 a^{2} R^{2}\right) .
\end{aligned}
$$

Also, the redshift is defined by the relation

$z=(1-2 u)^{-\frac{1}{2}}-1$,

whence we obtain

$z=\frac{1}{\sqrt{1-2\left\{\frac{1}{4} a r+\frac{26}{9} a^{2} r^{2}+\frac{130}{9} a^{3} r^{3}-\frac{a R}{18}\left(9+156 a R+1040 a^{2} R^{2}\right)\right\}}}$

The variations of the compactness and the redshift are shown in Fig. 5.

\subsection{Minimum mass-radius relation}

Buchdahl's condition is $\frac{m(R)}{R}<\frac{4}{9}<0.44$. Table 2 shows that $\frac{m(D)}{D}=0.22$ for the compact star Her X-1. So Buchdahl's condition is satisfied in our model. Also the value of the surface redshift for this star is 0.19 . We show the mass-radius ratios for some other stars also in Tables 1 and 2.

In Tables 1 and 2 the following symbols are used: $R_{\text {obs }}$ $=$ observed radius, $D_{\text {pre }}=$ predicted radius, $M_{R}=$ observed mass, $M_{D}=$ predicted mass, $M(D) / D=$ predicted massradius ratio, and $Z(D)=$ predicted surface redshift. Here the data are considered from Ref. [25].

\section{Conclusion}

First in our concluding remarks and discussions we would like to make some notes on observations regarding the radial pressure, which is expected to be positive at the boundary $r=$ 
Table 1 Comparative study of the physical parameters for compact star and presented model

\begin{tabular}{lclll}
\hline Compact stars & $R_{\text {obs }}(\mathrm{km})$ & $D_{\text {pre }}(\mathrm{km})$ & $M_{R}\left(M_{\odot}\right)$ & $M_{D}\left(M_{\odot}\right)$ \\
\hline Her $X-1$ & 6.70 & 6.788 & 0.9824 & 1.02 \\
$R X J 1856-37$ & 6.00 & 6.08 & 0.9042 & 1.03 \\
$S A X J(S S 1)$ & 7.07 & 7.08 & 1.4349 & 1.4071 \\
$S A X J(S S 2)$ & 6.35 & 6.38 & 1.3237 & 1.2970 \\
$P S R J 1614-2230$ & 10.3 & 10.37 & 1.97 & 1.96
\end{tabular}

Table 2 Comparative study of the physical parameters for compact star and presented model

\begin{tabular}{llccc}
\hline Compact stars & $a\left(\mathrm{~km}^{-1}\right)$ & $R(\mathrm{~km})$ & $M(D) / D$ & $Z(D)$ \\
\hline Her X -1 & -0.01860326 & 10 & 0.22 & 0.19 \\
$R X J 1856-37$ & -0.02208016 & 8.7 & 0.24 & 0.22 \\
SAX J $(S S 1)$ & -0.02070156 & 9.7 & 0.29 & 0.28 \\
SAX J $(S S 2)$ & -0.02322041 & 8.8 & 0.29 & 0.29 \\
PSR J $1614-2230$ & -0.01375931 & 14.4 & 0.27 & 0.26 \\
\hline
\end{tabular}

$10 \mathrm{~km}$. However, the tangential pressure in the present model actually becomes zero at $r=6.788 \mathrm{~km}$. Also we observe that the stability condition by the Herrera approach [24] is well satisfied within the radius $r=6.788 \mathrm{~km}$. Therefore, with reference to these observations, we can think of the formation of our stellar model of two phases. In phase I there is a shell of $2.3 \mathrm{~km}$ forming the outer region of the star whereas the phase II consists of a core of radius $6.7 \mathrm{~km}$ forming the inner region of the star. Now we observe from the related graphs that all the features of the star are physically valid within the core but some of the features do not show physical validity beyond the core i.e. within the outer shell. It is interesting to note that we find a well-known star, $\operatorname{Her} X-1$ of radius 6.7 $\mathrm{km}$, which can be explained by our model very well if we take only the core part of the star into our physical consideration.

For the present model of radiating compact stars as described above we observe the following interesting and salient features:

(1) We obtain a general mass function by solving a highly nonlinear equation of the mass by the HPM. In the HPM the homotopy structure consists of a linear and a nonlinear functional part. The choices of these functional operators are such that the trivial problem (the linear part) is continuously deformed to the original problem with variation of the embedding parameter $\in$ from 0 to 1 . In the present study, however, we use a particular homotopy structure to solve the nonlinear differential equation for the mass function so that all results obtained in this paper are valid subject to an approximation given by this particular homotopy.

(2) For $n=0$ the mass has no dependence on the radial distance. Again for $n=1$ the mass function becomes indeterminate. So we start our investigation with $n=2$.
(3) We find that the tangential pressure becomes negative throughout the whole region for the different groups of stars ( $E, M, K, G$ type). However, in the literature we note that this type of special solutions could exist [26].

(4) The features of the compact star of radius $6.788 \mathrm{~km}$ are well explained. We figure out that the mass of the star is 1.02 solar mass. This matches the observed compact star Her $X-1$ of radius $6.7 \mathrm{~km}$ and a mass of 0.9824 solar mass. Some other compact stars are also shown in Tables 1 and 2.

(5) For $n=3$, as a particular case of the present paper, we have already shown that the model supports a brown dwarf of $E 0$ type rather than compact stars. Also we have shown there that the brown dwarf is partially stable [21].

Regarding the MEP we have made some specific observations:

(1) Based on the work by Rahaman et al. [21] we note that there are several papers available in the literature where one may find the application of the MEP in the anisotropic case. In this connection a comment by Richstone and Tremaine [27] seems convincing: "It is sometimes argued that in maximum-entropy models the velocity-dispersion tensor must be isotropic and hence the distribution function must be a function of energy alone. In fact this is not correct when we seek the maximum-entropy model subject to the constraint of a measured density profile. In this case, might gradients in the dispersion cause anisotropy in the dispersion tensor of a maximum-entropy distribution function" Thus, as a future plan we would like to consider the anisotropic case under the MEP as has been performed by several researchers under different requirements [28-30]. However, applications of the HPM can 
be found in the field of astrophysics in different contexts paving the way to a new research arena $[31,32]$.

(2) Rahaman et al. [21] argued that instability may be an inherent property of any radiating compact stars under the HPM and the MEP as observed by them with brown dwarf stars. Instability has also been observed in the extension of the work of Bhar et al. [33], related to highly compact stars such as neutron stars and strange stars. In the present model we also observe the same feature of instability and may conclude that the applicability of the HPM and the MEP to radiating and highly compact stars may have some intrinsic problem to provide the entire physical validity of the models.

Acknowledgments FR and SR are thankful to the authority of InterUniversity Centre for Astronomy and Astrophysics, Pune, India, for providing them a Visiting Associateship under which a part of this work was carried out.

Open Access This article is distributed under the terms of the Creative Commons Attribution 4.0 International License (http://creativecomm ons.org/licenses/by/4.0/), which permits unrestricted use, distribution, and reproduction in any medium, provided you give appropriate credit to the original author(s) and the source, provide a link to the Creative Commons license, and indicate if changes were made. Funded by SCOAP ${ }^{3}$.

\section{References}

1. J.H. He, Commun. Nonlinear Sci. Numer. Simul. 2, 230 (1997)

2. J.H. He, Comput. Methods Appl. Mech. Eng. 178, 257 (1999)

3. J.H. He, Int. J. Non-Linear Mech. 35, 37 (2000)

4. J.H. He, Int. J. Nonlinear Mech. 35, 115 (2000)

5. J.H. He, Appl. Math. Comput. 151, 287 (2004)

6. J.H. He, Int. J. Nonlinear Sci. Numer. Simul. 6, 207 (2005)

7. J.H. He, Phys. Lett. A 350, 87 (2006)

8. J.H. He, Therm. Sci. 2, 565 (2010)

9. S.J. Liao, The proposed homotopy analysis technique for the solution of nonlinear problems. Ph.D. thesis, Shanghai Jiao Tong University (1992)
10. G.L. Liu, New research directions in singular perturbation theory: artificial parameter approach and inverseperturbation technique. Conf. of 7th modern mathematics and mechanics, Shanghai, pp. 47-53 (1997)

11. S.J. Liao, Beyond Perturbation: Introduction to the Homotopy Analysis Method (Chapman \& Hall/CRC Press, Boca Raton, 2003). ISBN: 1-58488-407-X

12. S.J. Liao, Appl. Math. Comput. 147, 499 (2004)

13. S.J. Liao, Homotopy Analysis Method in Nonlinear Differential Equation (Springer, Berlin, 2012). ISBN: 978-3642251313

14. D.R. Finkelstein, Int. J. Theor. Phys. 47, 534 (2008)

15. A. Demir, S. Erman, B. Ozgur, E. Korkmaz, Bound. Value Probl. 2013, 61 (2013)

16. S.S. Siddiqi, M. Iftikhar, J. Asso, Arab. Univ. Bas. Appl. Sci. 18, 60 (2015)

17. V. Shchigolev, Univ. J. Appl. Math. 2, 99 (2014)

18. S.S. Nourazar, M. Soori, A. Nazari-Golshan, J. Appl. Math. Phys. 2015, 285 (2015)

19. F. Rahaman, K. Chakraborty, P.K.F. Kuhfittig, G.C. Shit, M. Rahman, Eur. Phys. J. C 74, 3126 (2014)

20. D. Deb, S. Roy Chowdhury, S. Ray, F. Rahaman. arXiv:1509.00401

21. F. Rahaman, S. Ray, A. Aziz, S. Roy Chowdhury, D. Deb. arXiv: 1504.05838

22. R.D. Sorkin, R.M. Wald, Z.J. Zhang, Gen. Relativ. Gravit. 13, 1127 (1981)

23. G.W. Gibbons, M.J. Perry, Proc. R. Soc. Lond. Ser. A 358, 467 (1978)

24. L. Herrera, Phys. Lett. A 165, 206 (1992)

25. S.K. Maurya, T.T. Smitha, Y.K. Gupta, F. Rahaman. arXiv: 1512.01667 [gr-qc]

26. F. Rahaman, M. Kalam, S. Chakraborty, K. Maity, B. Raychaudhuri. arXiv:0808.1626

27. D.O. Richstone, S. Tremaine, Astrophys. J. 327, 82 (1988)

28. H.J. Pirner, K. Reygers, B.Z. Kopeliovich, Phys. Rev. C 93, 034910 (2016)

29. T.K. Nakamura, Eos. Trans. AGU 80, 46 (2000)

30. M. Gedalin, Phys. Fluids B Plasma Phys. 3, 2149 (1991)

31. V.K. Shchigolev, Univ. J. Comput. Math. 3, 45 (2015)

32. V.K. Shchigolev. arXiv:1511.07459 [gr-qc]

33. P. Bhar, F. Rahaman, S. Ray, V. Chatterjee, Eur. Phys. J. C 75, 190 (2015) 